\title{
Математическое моделирование и расчет процесса деминерализации воды в промышленном фильтре Сообщение 4. Применение программы «Createscheme» для расчета циклического процесса деминерализации воды
}

\author{
Комарова И.В., Галкина Н.К., Прудковский А.Г., Хамизов Р.Х.
}

Институт геохимии и аналитической химии им. В.И.Вернадского РАН, Москва

Поступила в редакцию 25.12.2016 г.

\begin{abstract}
Рассмотрен циклический процесс сорбции, взрыхления, регенерации и отмывки в промышленном катионитовом фильтре центральной обессоливающей установки Новочеркасской ГРЭС при его длительной эксплуатации. Проведен расчет всех стадий процесса по программе "Createscheme" для каждого цикла. Для одного из 18 экспериментально исследованных циклов представлены расчетные и экспериментальные выходные кривые для иона натрия в середине слоя и на выходе из фильтра. Показано удовлетворительное совпадение расчетного и определенного экспериментально количества колоночных объемов очищенной воды.

Совпадение на всех стадиях процесса экспериментальных и расчетных данных свидетельствуют об адекватности предложенных математических моделей промышленному процессу водоподготовки.

Ключевые слова: циклический ионообменный процесс, взрыхление, регенерация, отмывка, очистка на частично регенерированном катионите, выходные кривые, программа «Createscheme».

\section{Mathematical simulation and calculation of the process of water demineralization in industrial filter Part 4. Using the program «Createscheme» for the computation of water demineralization cyclic process}

\author{
Komarova I.V., Galkina N.K., Prudkovskii A.G., Khamizov R.Kh. \\ Vernadsky Institute of Geochemistry and Analytical Chemistry of Russian Academy of Sciences \\ (GEOKHI RAS). Moscow
}

The aim of the work was to study complete cycles of H-cationic water treatment by the methods of computation and industrial scale experimentation on one of the operating filters at the Novocherkassk Power Plant central desalting unit.

The work was performed numerical experiments with the «Createsheme» program, based on the model of a multi-component sorption dynamics. The distinctive property of the model was the possibility of accounting the variable boundary conditions like the composition of raw water. The model parameters were: ion exchange equilibrium constants and diffusion kinetic coefficients, previously defined by the authors.

Industrial scale experiments were carried out on a filter with cation exchanging resin KU-2. Breakthrough curves for cations were recorded in course of 18 technological cycles including for each one the stages of water purification on the partially regenerated cation-exchanger, filter regeneration with sulphuric acid, washing with water. The concentration curves for hydrogen were also obtained for the stages of regeneration. For some cycles, backwashing stages were analysed using the resin samples taken at different points 
of the filter. It was approved by the comparison of theoretical results and experimental data that the mathematical model of the dynamics of a multicomponent exchange, forming the basis of the proposed computation program, takes into account technological features and adequately describes the industrial process of ion exchange demineralization of water.

Keywords: Cyclic ion exchange process, backwashing, regeneration, washing, repeated sorption process on partly regenerated sorbent, break-through curves, computer program «Createscheme».

\section{Введение}

В предыдущих сообщениях была рассмотрена гидродинамика стадий регенерации и отмывки катионита, влияние изменения входных параметров и влияние взрыхления на процесс очистки воды на катионитовом фильтре первой ступени центральной обессоливающей установки Новочеркасской ГРЭС.

В развитие ранее проводимых работ по моделированию и оптимизации процесса деминерализации воды для ТЭС и АЭС [1], на основе созданной программы «Createscheme»[2] проведен расчет циклического процесса очистки воды, включающего стадии взрыхления, регенерации, отмывки и сорбции на частично регенерированном катионите КУ-2 в промышленном фильтре первой ступени схемы НГРЭС.

Применение программы для расчета проведенного нами ранее промышленного эксперимента позволило сопоставить расчетные и экспериментальные данные и определить основные технологические характеристики процесса: количество и качество очищенной воды, режимы взрыхления, регенерации и отмывки.

\section{Эксперимент}

При проведении расчетов в качестве исходного состояния системы использовали экспериментальное распределение компонентов по слою катионита в одном из рабочих циклов перед началом опытов.

Каждый эксперимент рассматривался как последовательность стадий технологического процесса, включающего неполную регенерацию, отмывку осветленной водой, очистку воды от катионов до проскока натрия, взрыхление фильтра.

\section{Обсуждение результатов}

Сравнение результатов расчетов с технологическими данными проводили по сопоставлению выходных кривых иона натрия в момент останова фильтра на регенерацию, поскольку ион натрия определяет качество очищенной воды.

В данном сообщении приводятся экспериментальные и расчетные данные для одного из опытов, проведенных с взрыхлением.

На рис.1 показано соответствие экспериментальной выходной кривой регенерации натрия после взрыхления с расчетной выходной кривой, полученной при условии полного перемешивания во время взрыхления. Как следует из полученных данных, расчетные и экспериментальные выходные кривые достаточно хорошо совпадают.

На рис.2 приведены расчетные и экспериментальные выходные кривые отмывки катионита осветленной водой от серной кислоты после регенерации. Поскольку отмывка проводится осветленной водой, глубина отмывки определяется катионным составом отмывочной воды, поэтому увеличение времени отмывки не приводит к улучшению качества отмывки.

Комарова и др. / Сорбционные и хроматографические процессы. 2017. Т. 17. № 1 

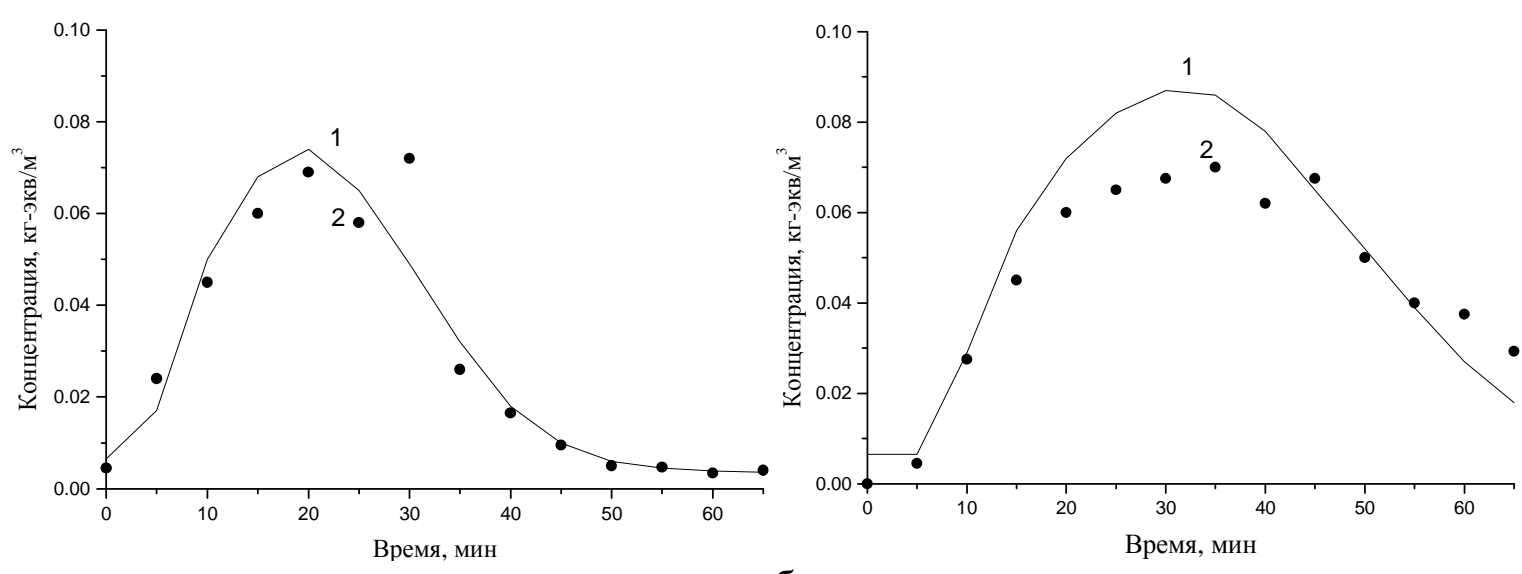

а 6

Рис. 1. Расчетные и экспериментальные выходные кривые регенерации натрия в середине слоя - (а) и на выходе из фильтра - (б) 1 - расчет 2 - эксперимент

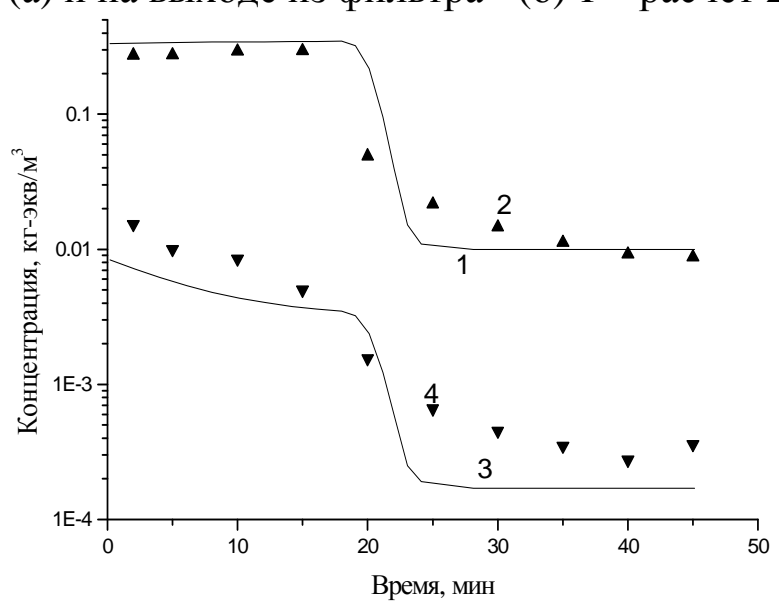

Рис. 2. Выходные кривые отмывки катионита от серной кислоты и натрия на выходе из фильтра: водород: 1 - расчет 2 - эксперимент; натрий: 3 - расчет, 4 - эксперимент

На рис.3 приведено рассчитанное по программе распределение всех компонентов по слою катионита после регенерации и отмывки. Как следует из рисунка 3 , в результате регенерации натрий практически десорбирован из катионита, а верхние слои катионита частично регенерированы по двухзарядным ионам. При отмывке происходит дополнительная регенерация катионита за счет кислоты, которая вытесняется из пространства над слоем катионита, и кислоты, оставшейся между зернами катионита. На рисунке видно, что использование осветленной воды при отмывке катионита приводит к частичной отработке верхних слоев катионита по натрию, магнию и кальцию.

На рис. 4 приведены расчетные и экспериментальные выходные кривые иона натрия в среднем сечении слоя и на выходе из фильтра на стадии очистки воды. В связи с работой водоподготовительной установки в реальном режиме фильтр отключался на регенерацию при заданном проскоке натрия, равным $1.1510^{-3}$ кг-экв/ $\mathrm{m}^{3}$. Видно, что в момент проскока натрия на выходе из фильтра концентрация натрия в середине фильтра достигает максимальной концентрации, равной суммарной концентрации катионов, содержащихся в осветленной воде. Из приведенных выходных кривых видно, что расчетные и экспериментальные данные удовлетворительно совпадают. 


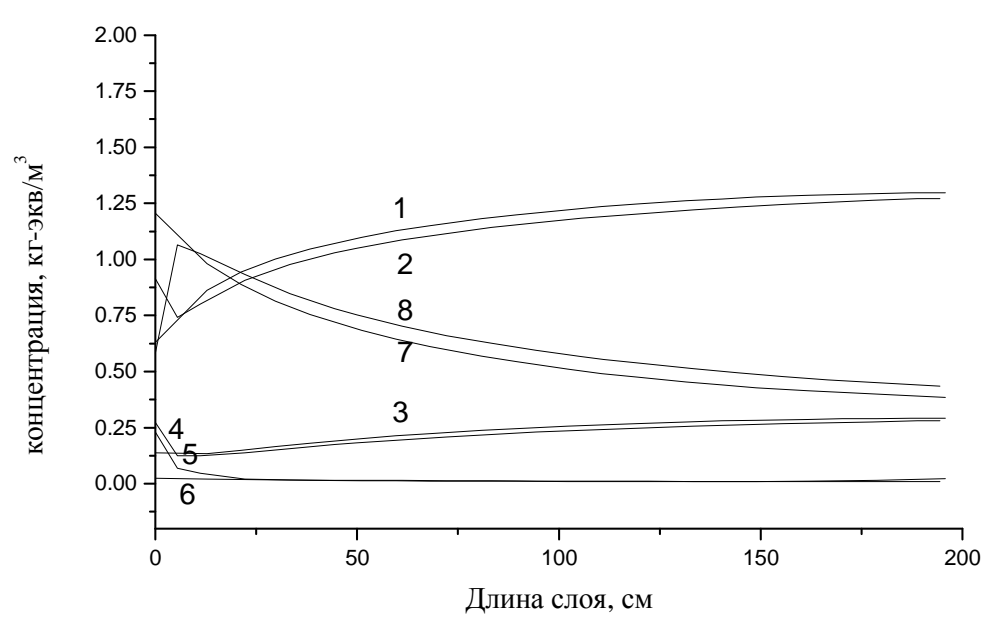

Рис. 3. Расчетное распределение катионов по слою катионита после регенерации: 1- кальций 3- магний 5- натрий 7- водород; после отмывки: 2- кальций 4- магний 6- натрий 8- водород
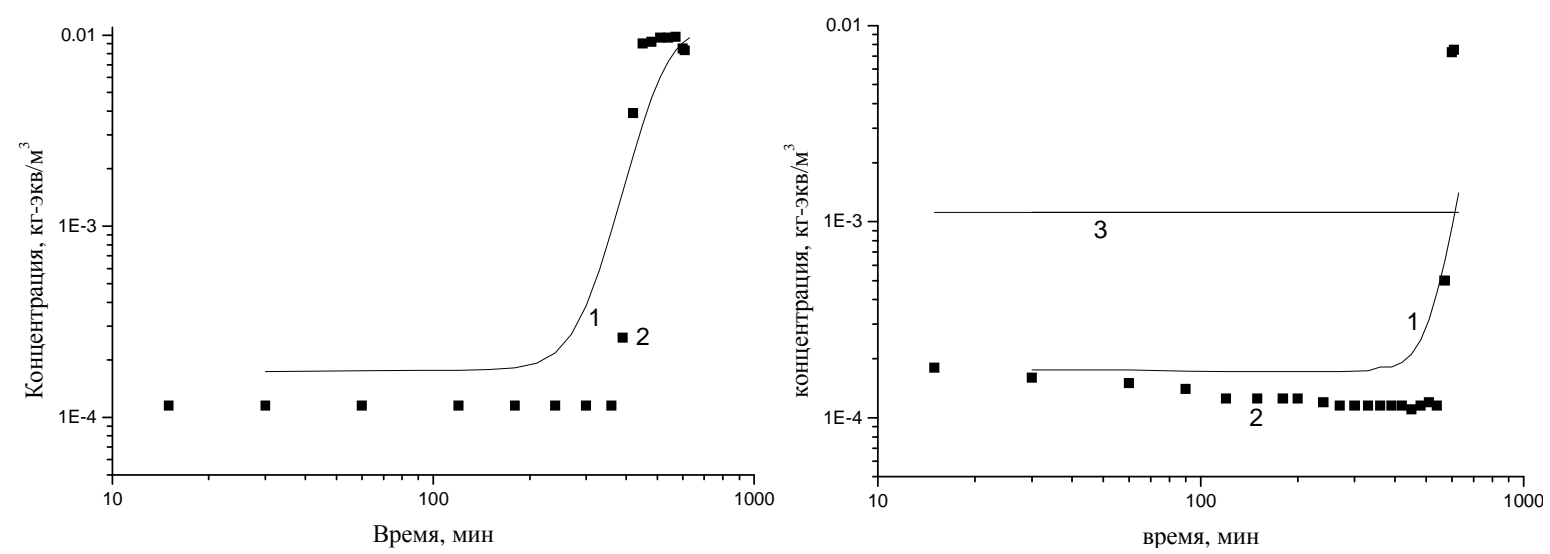

Рис. 4. Выходные кривые сорбции натрия в середине слоя - (а) и на выходе из фильтра - (б): 1 - расчет 2 - эксперимент 3 - концентрация натрия при проскоке

При очистке воды на частично регенерированном катионите концентрация натрия в начале рабочего цикла в данном опыте составляет $1.810^{-4} \mathrm{\kappa г-экв/ \textrm {M } ^ { 3 }}$ за счет вытеснения из замыкающих слоев катионита иона натрия, оставшегося после регенерации и отмывки катионита. Концентрация натрия затем нарастает и на выходе из фильтра достигает заданной величины проскока.

На рис. 5 представлено распределение ионов по слою катионита после рабочего цикла. Распределение компонентов по слою катионита после рабочего цикла объясняет, почему в проведенных экспериментах на промышленном фильтре время до проскока натрия на частично регенерированном катионите существенно меньше, чем на катионите в водородной форме. После регенерации разбавленной серной кислотой и последующей отмывки значительная часть слоя находится в кальциевомагниевой форме. Поэтому в процессе очистки наименее сорбируемый ион натрия сорбируется частично на водородной форме, а в основном на кальциевой и магниевой. В этом случае сорбция реализуется по вогнутой изотерме, что приводит к короткому циклу.

В качестве одного из показателей совпадения расчета с промышленным экспериментом рассматривалось количество получаемой в каждом из циклов Нкатионированной воды (таблица 1). 


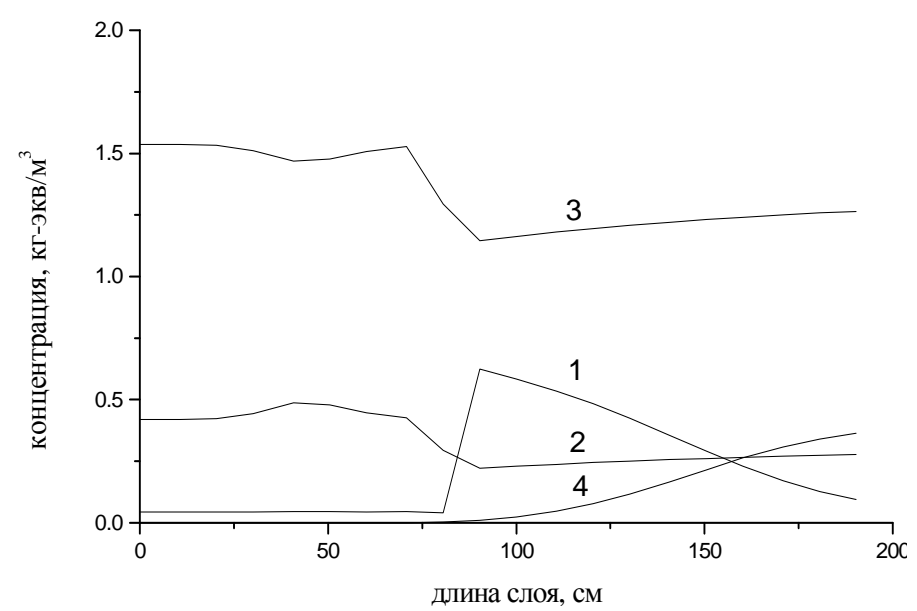

Рис. 5. Расчетное распределение катионов по слою катионита после сорбции 1 - натрий, 2 - магний, 3 - кальций, 4 - водород

Таблица 1. Сравнение экспериментального и расчетного количества очищенной воды

\begin{tabular}{|c|c|c|c|}
\hline $\begin{array}{c}\text { Рабочий } \\
\text { цикл }\end{array}$ & $\begin{array}{c}\text { Скорость потока } \\
\mathrm{m}^{3} / \text { час }\end{array}$ & \multicolumn{2}{|c|}{$\begin{array}{c}\text { Количество очищенной воды, } \\
\text { колоночные объемы }\end{array}$} \\
\hline 1 & & 52 & расчет \\
\hline 2 & 74 & 55 & 50 \\
\hline 3 & 73 & 36 & 51 \\
\hline 4 & 61 & 39 & 47 \\
\hline 5 & 80 & 45 & 42 \\
\hline 6 & 74 & 34 & 47 \\
\hline 7 & 75 & 50 & 52 \\
\hline 8 & 70 & 50 & 49 \\
\hline 9 & 77 & 53 & 50 \\
\hline 10 & 109 & 47 & 58 \\
\hline 11 & 98 & 49 & 50 \\
\hline 12 & 89 & 55 & 49 \\
\hline 13 & 89 & 59 & 49 \\
\hline 14 & 107 & 50 & 49 \\
\hline 15 & 115 & 47 & 41 \\
\hline 16 & 82 & 46 & 45 \\
\hline 17 & 117 & 49 & 45 \\
\hline 18 & 143 & 45 & 50 \\
\hline
\end{tabular}

В результате сравнения расчетов по программе и экспериментальных данных показано, что математическая модель каждой стадии процесса деминерализации воды в промышленном фильтре адекватно описывает реальный процесс.

\section{Заключение}

На основе послойной математической модели разработаны и апробированы алгоритмы и программа расчета и визуализации полных циклов процессов сорбционной очистки воды и регенерации катионита, позволяющие учитывать переменные граничные условия. Полученные результаты позволяют также применить предло- 
женные и проверенные в реальных условиях математические модели при разработке системы контроля и управления процесса ионообменной деминерализации воды.

За счет быстрого получения информации о текущем состоянии технологического процесса, полученного в результате расчета, по сравнению с экспериментом, можно менять режим работы фильтра на любой стадии при изменении исходных данных, например при изменении состава очищаемой воде или производительности, остановить фильтр на регенерацию, не допустив при этом проскока целевого компонента.

Все разработанные модели включены в единый вычислительный комплекс программ «Createscheme», способный моделировать технологические стадии процесса деминерализации воды, а также совместную работу произвольного числа фильтров с произвольным их соединением.

Таким образом, алгоритм, отлаженный на отдельном изолированном фильтре, в настоящее время готов для применения в реальных сложных технологических схемах.

\section{Список литературы}

1. Комарова И.В., Галкина Н.К., Рубинштейн Р.Н., Сенявин М.М.// Журнал физической химии. 1973. Т. 47. № 1. С.124-129

\section{References}

1. Komarova I.V., Galkina N.K., Rubinshtejn R.N., Senjavin M.M., Zhurnal fizicheskoj himii, 1973, Vol. 47, No 1, pp. 124-129.

Комарова Ирина Владимировна к.Х.н., старший научный сотрудник, лаборатория сорбционных методов, ГЕОХИ РАН

Галкина Надежда Константиновна - к.х.н., старший научный сотрудник, лаборатория сорбционныХ методов, ГЕОХИ РАН

Прудковский Андрей Гаральдович - д.ф-м.н., старший научный сотрудник, лаборатория сорбционных методов, ГЕОХИ РАН,

Хамизов Руслан Хажсетович - Д.Х.н., Зав. лаборатории сорбционных методов, ГЕОХИ РАН
2. Прудковский А.Г., Комарова И.В., Галкина Н.К. // Сорбционные и хроматографические проиессы. 2014. Т. 14. № 5. С. 824-831
2. Prudkovskii A.G., Komarova I.V., Galkina N.K. Sorbtsionnye i khromatograficheskie protsessy, 2014, Vol. 14, No 5, pp.824-831.

Komarova Irina V. - Ph.D. (Chem.), Senior researcher, Lab of Sorption Methods, GEOKHI RAS

Galkina Nadezda K. - Ph.D. (Chem.), Senior researcher, Lab. of Sorption Methods, GEOKHI RAS

Prudkovskii Andrei G. - Dr.Sci. (Phys/Math.), Leading researcher, Lab of Sorption Methods, GEOKHI RAS

Khamizov Ruslan Kh. Dr.Sci.(Chem), Head of the Laboratory of Sorption Methods, GEOKHI RAS 